\title{
AN EVIDENCE MULTICOPY DYNAMIC DATA POSSESSION IN MULTI CLOUD COMPUTING SYSTEM
}

\author{
V.Sri Vidya ${ }^{1}$, R. Pavan Kumar ${ }^{2}$
}

\begin{abstract}
Presently a day more associations expanding and are picking outsource information to remote cloud services provider. The customers can lease the CSPs storage infrastructures to stores and recovers practically boundless measure of information by paying charges metered in gigabyte/month. In this paper, proposes a map-based provable multicopy dynamic data possession $(M B-P M D D P)$ scheme that is needs to takes after elements: 1) it's give a confirmations to customer that the CSPs is not storing so as to go to cheat the duplicate of information; 2) its provide an outsourcing of element information and its support block levels operation, for example, piece adjustments, insertion, cancellation, and annex; and 3) it permits authorized users to consistently get to the record duplicates put away by the CSP. It gives a near examination of the proposed MB-PMDDP plan with a reference model acquired by amplifies existing provables belonging of elements single duplicates plan.
\end{abstract}

Keywords—Cloud computing, Data replications, Outsourcing data storages, Dynamic Environments.

\section{INTRODUCTION}

Presently a day's Outsourcing information to a remote cloud administration suppliers permits association to stores more information on the CSP than on private PC frameworks. Such outsourcing of data storages enable organizationsto focuses on development and soothe the weight of steady server overhauls and other registering issues. The privacy issue can be taken care of by scrambling touchy information before outsourcing to remote server. All things considered, its crucials requests of client to have solid confirmation that the cloud servers still have their information and it's being tamparated with or mostly erase over times. Therefore, numerous specialists have concentrated on the issue of provable data possessions (PDP) and we propose distinctive techniques to review the information put away on remote servers.

Hypothesis of a Paper: PDP is a method for accepting information trustworthiness over remote servers. In an ordinary PDP model, the information proprietor produces some $\mathrm{MD} /$ data for an information records to be utilized laters for confirmations purposes through a difficulties reaction conventions with the remotes cloud servers. The proprietor sends the record to be put away on a remote server which might have been untrustes, and erases the neighborhood duplicate of the documents. One can be the first information proprietor or a trusted substance that imparts some data to the proprietor [1]-[9]. One of the center configuration standards of outsourcing information is to give dynamic conduct of information to various applications.

PDP plans introduced [1]-[9] concentrate on just static or warehoused information that outsource information's is kept unaltered over remote servers and PDP developments that arrangement with element information are [10]-[14]. PDP plans have been exhibited for numerous duplicates of static information [15]-[17], to the best of learning; this works is the principal PDPs plots specifically managing products duplicates of element information. While confirming numerous information duplicates and the general frameworks trustworthiness checks comes up short if there are one or more debased duplicates. To address this issue and perceive which duplicates have been defiled, in this paper examines a slight change to be connected to the proposed scheme.

\section{FUNDAMENTAL COMMITMENTS}

Our commitments can be abridged as takes after:

- It proposes of a map-based provables multicopy dynamics data possession (MB-PMDDP) schemes, gives a sufficient surety that the CSP stores all duplicates that are settled upon in the administration contract and the plan bolsters outsourcing of element information.

- It gives intensive examinations of MB-PMDDP with a reference plan, which one can acquire by broadening existing PDP models for element single-duplicate information.

- The paper demonstrates the security of plan against intriguing servers, and examines proposed plan to distinguish tainted duplicates.

Framework Design: The distributed computing storages models considered in this work comprises of three primary parts as represented in Fig.

1. An information proprietor that can be an association initially having delicate information to be put away in the cloud;

2. CSP who oversees cloud servers (CSs) and gives paid storage room on its base to store the proprietor's records;

3. Authorized client's arrangement of proprietor's customers who have the privilege to get to the remotes information. 
The storages models utilized as a part of this works can be adoptes by numerous reasonable applications.

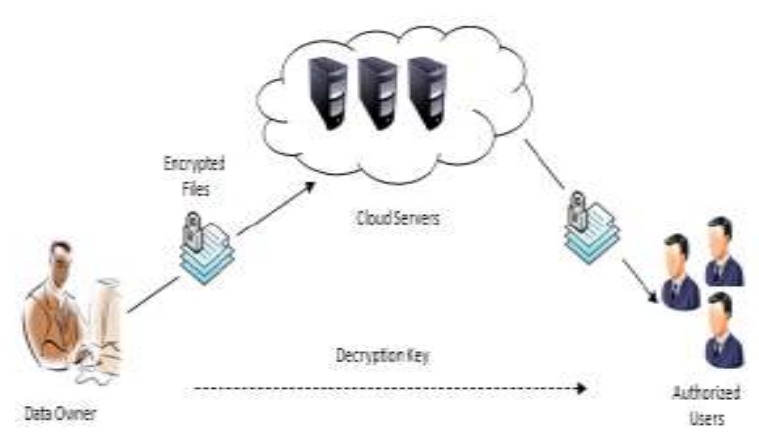

The quantity of duplicates rely on upon the natures of information's more duplicates are required for basic information that can't undoubtedly be imitated, and to accomplish a larger amount of adaptability. This basic information ought to be repeated on different servers over various server farms. The CSP estimating model is identified with the quantity of information duplicates.

An approved client of the outsourced information sends an information access solicitation to the CSP and gets a document duplicate in a scrambled structure that can be unscrambled utilizing a mystery keys offer with the proprietors. By burdens adjusting component utilized by the CSP to arrange the work of the servers, the information access solicitations is immediate to the server with the most reduced blockage, and hence the client doesn't know about which duplicate has been gotten.

The Threat Model: The integrity of customers' information in the cloud might be at threats because of the accompanying reasons. To start with The CSP whose objectives is similar to make benefit and keep up a notorieties has an expanding, and it has a discarding so as to motivate forces to shrouds information misfortunes stockpiles information's that has not been or is once in a while got to.

Second, a dishonestes CSP might stores less duplicates than what has been concurs upon in the administrations contacts with are accurately put away arraigns.

Third to spare the computational asset, the CSP might thoroughly disregard the information upgrade demands issued by the proprietors, or not executes them on all duplicates prompting irregularity between the document duplicates. The objective of the proposed plan is to identify the CSP bad conduct by accepting the number and respectability of document duplicates.

\section{PROPOSED MB-PMDDP PLAN}

Producing novel differentiable duplicates of the information document are the center to plan a provable multicopy information belonging plans. Indistinguishably duplicate empowers the CSPs to just trick the proprietors by putting away one and only duplicate and imagines that it stores products duplicates. Utilizing a basic yet productive ways, the propose plan create uniqueness duplicate use the dispersions property of any Secures encryptions plans. The communications between the approve client and the CSPs is consider through this procedures of create particular duplicates, where the formers can decodes/access a documents duplicates gets from the CSPs. In the propose plans, the approved client needs just to keep a singles privileged insights key to decodes the documents duplicates, and it is not as a matter of course to perceives the lists of the gets duplicates.

MB PMDDPs plans permits the information proprietors to upgrades and scales the piece of records duplicates outsource to cloud servers which might be untrusted. Accepting such duplicates of progress information required the learning of the square of renditions to guarantee that the information's pieces in all duplicate are predictable with the latest change issue by the proprietors. In addition, the checked ought to know about the pieces files to ensures that the CSPs has embeds or included the news hinders at the solicitations position in all duplicates. To this finishes, the propose plans depends on utilizing a little information's structures, which we call a guide adaptations

\section{IMPLEMENTATION}

The paper executed the proposed MB-PMDDP plan and the TB-PMDDP reference model on top of Amazon S3 is a web storages services to stores and recovers practically boundless measures of data's. In addition, it empower client to determine geologies area for putting away theirs data's. It introduces a plan comprises of 3 modules:

OModules (proprietors modules), CModules (CSPs modules), and VModules (verifiers modules). OModules, which are running on the proprietor's sides, is a library that incorporates KeyGens, CopyGens, TagGens, and Gets ready Upgrades of calculation. CModules is a library that keeps running on Amazons EC2 and incorporates Executes Overhauls and Demonstrates calculation. VModules is a library to be keep running at the verifiers sides and its incorporate the calculations and propose another PDPs plans, which its bolster outsourcing of various duplicates and element information, the information proprietors is equipped for not just filing and getting to the information's duplicates stores by CSPs, furthermore redesigns and scaling these duplicates on the remotes servers.

\section{CONCLUSION}

The propose MB PMDDPs plans outflank the TB-PMDDPs approaches gets from a classes of progress single-duplicates PDPs model. The TB-PMDDPs prompts high stockpiles overheads on the remotes server and high calculation on both the CSPs and the verifier's side. The MB-PMDDPs plans huge decrease the calculations times amid the difficulties reactions stages which make it more reasonable for application, for a bigger quantities of verifier are 
associates with the CSPs bringing on a colossal calculations over heads on the server. Plus, it has lower stockpiles over heads on the CSPs, and therefore decreases the charge paid by the cloud client. A light changes can be dones on the propose plans to underpins the components of recognizing the indice of corruptness duplicates. The undermines information duplicates can be remake even from a finishes harms utilizing copy duplicates on others server. It has shown toss security examination, that the proposed plan is provably secure.

\section{REFERENCES}

[1]. G. Ateniese et al., "Provable data possession at untrusted stores," inProc. 14th ACM Conf. Comput. Commun. Secur. (CCS), New York, NY,USA, 2007, pp. 598-609.

[2]. K. Zeng, "Publicly verifiable remote data integrity," in Proc. 10th Int.Conf. Inf. Commun. Secur. (ICICS), 2008, pp. 419-434.

[3]. Y. Deswarte, J.-J. Quisquater, and A. Saïdane, "Remote integritychecks," in Proc. 6th Working Conf. Integr. Internal Control Inf.Syst. (IICIS), 2003, pp. 1-11. 